\title{
Genomic analysis demonstrates that histologically-defined astroblastomas are molecularly heterogeneous and that tumors with MN1 rearrangement exhibit the most favorable prognosis
}

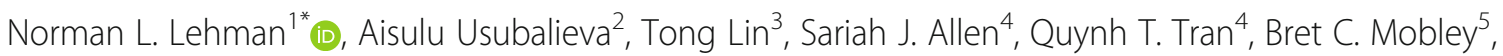

Roger E. McLendon", Matthew J. Schniederjan, Maria-Magdalena Georgescu ${ }^{8}$, Marta Couce ${ }^{9}$, Mohanpal S. Dulai ${ }^{10}$, Jack M. Raisanen ${ }^{11}$, Mousa Al Abbadi ${ }^{12}$, Cheryl A. Palmer ${ }^{13}$, Eyas M. Hattab ${ }^{1}$ and Brent A. Orr ${ }^{4^{*}}$

\begin{abstract}
Astroblastoma (AB) is a rare CNS tumor demonstrating abundant astroblastomatous pseudorosettes. Its molecular features have not been comprehensively studied and its status as a tumor entity is controversial. We analyzed a cohort of 27 histologically-defined ABs using DNA methylation profiling, copy number analysis, FISH and sitedirected sequencing. Most cases demonstrated mutually exclusive MN1 rearrangements $(n=10)$ or BRAF $F^{V 600 E}$ mutations $(n=7)$. Two additional cases harbored RELA rearrangements. Other cases lacked these specific genetic alterations $(n=8)$. By DNA methylation profiling, tumors with MN1 or RELA rearrangement clustered with highgrade neuroepithelial tumor with MN1 alteration (HGNET-MN1) and RELA-fusion ependymoma, respectively. In contrast, $B R A F^{V 600 E}$-mutant tumors grouped with pleomorphic xanthoastrocytoma (PXA). Six additional tumors clustered with either supratentorial pilocytic astrocytoma and ganglioglioma (LGG-PA/GG-ST), normal or reactive cerebrum, or with no defined DNA methylation class. While certain histologic features favored one genetic group over another, no group could be reliably distinguished by histopathology alone. Survival analysis between genetic $A B$ subtypes was limited by sample size, but showed that MN1-rearranged AB tumors were characterized by better overall survival compared to other genetic subtypes, in fact, significantly better than $B R A F^{V 600 E}$-mutant tumors $(P=0.013)$. Our data confirm that histologically-defined $A B s$ are molecularly heterogeneous and do not represent a single entity. They rather encompass several low- to higher-grade glial tumors including neuroepithelial tumors with MN1 rearrangement, PXA-like tumors, RELA ependymomas, and possibly yet uncharacterized lesions. Genetic subtyping of tumors exhibiting $A B$ histology, particularly determination of $M N 1$ and $B R A F^{V 600 E}$ status, is necessary for important prognostic and possible treatment implications.
\end{abstract}

\footnotetext{
*Correspondence: nllehman@yahoo.com; brent.orr@stjude.org

'Department of Pathology and Laboratory Medicine, University of Louisville, Louisville, KY 40292, USA

${ }^{4}$ Department of Pathology, St. Jude Children's Research Hospital, 262 Danny

Thomas Place, Memphis, TN 38105, USA

Full list of author information is available at the end of the article
}

(c) The Author(s). 2019 Open Access This article is distributed under the terms of the Creative Commons Attribution 4.0 International License (http://creativecommons.org/licenses/by/4.0/), which permits unrestricted use, distribution, and reproduction in any medium, provided you give appropriate credit to the original author(s) and the source, provide a link to the Creative Commons license, and indicate if changes were made. The Creative Commons Public Domain Dedication waiver (http://creativecommons.org/publicdomain/zero/1.0/) applies to the data made available in this article, unless otherwise stated. 


\section{Introduction}

Astroblastomas (ABs) are rare glial neoplasms characterized by relatively compact growth and a predominantly perivascular tumor cell arrangement [1]. They are mostly superficial cerebral lesions presenting in the first to fourth decades of life, with some studies demonstrating a strong female predominance (reviewed by Aldape and Rosenblum [1]). ABs have not been assigned a specific World Health Organization (WHO) tumor grade. They are usually benign; however, clinically aggressive $A B$ cases have been reported $[1,15]$.

The ontologic and diagnostic significance of $\mathrm{ABs}$ has long been debated. Authors have variably argued that they are variants of diffuse astrocytoma or ependymoma [14]. We recently provided evidence that ABs may be related to pleomorphic xanthoastrocytomas (PXAs), based on overlapping clinicopathologic features and detection of the V600E mutation of the B-Raf serine-threonine kinase $\left(B R A F^{V 600 E}\right)$ in a subset of lesions [15].

MN1 (meningioma [disrupted in balanced translocation] 1) is a transcriptional coregulator important in development and is implicated in the pathogenesis of meningioma and acute myeloid leukemia $[9,16]$. A recent study of tumors diagnosed as CNS primitive neuroectodermal tumors (PNETs), identified four novel DNA methylation-defined brain tumor groups [19]. One group, termed high-grade neuroepithelial tumor with MN1 alteration (HGNET-MN1), showed recurrent rearrangements of the $M N 1$ gene, located at 22q12.3-qter. Reclustering of the DNA methylation data with tumors that did not carry a diagnosis of PNET revealed that approximately $40 \%$ of tumors clustering within the HGNET-MN1 DNA methylation class were institutionally diagnosed as AB [19]. Subsequent evaluation of limited cohorts confirmed that MN1 rearrangements occur in a subset of $A B s$ [10, 22].

The presence of $B R A F^{V 600 E}$ mutations in some $A B s$ and $M N 1$ rearrangements in others raises questions as to whether $\mathrm{AB}$ represents a distinct entity, or a histologic pattern exhibited by multiple glial tumor types. We, therefore, evaluated the molecular characteristics of 27 histologically-defined ABs using DNA methylation profiling, chromosomal copy number analysis, fluorescence in situ hybridization (FISH), and BRAF mutation analysis.

\section{Methods}

\section{Cases}

Construction of a clinical cohort of $28 \mathrm{AB}$ cases was previously described [15]. The published cohort was augmented for this study with six additional histologically-defined ABs following appropriate Institutional Review Board approval. Seven cases from the original cohort were excluded due to insufficient material for further analysis. This left 27 cases in the current cohort. Patient demographics and pathologic data are presented in Table 1. Cases from our original cohort were designated with the corresponding case numbers from that study [15] and included cases C1, C3, C5-C14, C16, C17, C19-C24, and C26. New cases were designated C29-C34. Recurrent tumor from two cases (designated rC10 and rC33) was available for limited analysis; thus, there were 29 total samples from 27 unique patients.

\section{BRAF $^{\mathrm{V} 600 \mathrm{E}}$ mutation analysis}

Tumor BRAF $F^{V 600 E}$ mutation status was tested using a single nucleotide extension assay followed by Sanger sequencing as previously described [15]. BRAF ${ }^{V 600 E}$ testing of the new samples was performed as previously described [4].

\section{Immunohistochemistry}

Immunohistochemical staining for olig2 and Ki-67 was performed as previously described [15].

\section{Fluorescence in situ hybridization}

Dual-color FISH was performed on $4-\mu \mathrm{m}$ paraffinembedded tissue sections. Break-apart probes for $M N 1$ were derived from BAC clones RP11-432I9 and RP11-736H16 (BACPAC Resources, Oakland, CA). Probes were labeled with either AlexaFluor-488 (green) or AlexaFluor-555 (orange-red) fluorochromes (Invitrogen, Carlsbad, CA) and validated on normal control metaphase spreads. BAC probe mixtures were diluted 2:50 in hybridization buffer and co-denatured with the target cells on a slide moat at $90^{\circ} \mathrm{C}$ for $12 \mathrm{~min}$. The slides were incubated overnight at $37^{\circ} \mathrm{C}$ on a slide moat followed by a $4 \mathrm{M}$ Urea/2xSSC wash at $25^{\circ} \mathrm{C}$ for $1 \mathrm{~min}$. Nuclei were counterstained with DAPI $(200 \mathrm{ng} / \mathrm{ml})$ (Vector Labs, Burlingame, CA) for viewing with an Olympus BX51 fluorescence microscope equipped with a 100 watt mercury lamp; FITC, Rhodamine, and DAPI filters; 100X PlanApo (1.40) oil objective; and a Jai CV digital camera. Images were captured and processed with an exposure time ranging from 0.1 to $2 \mathrm{~s}$ for each fluorochrome using Cytovision v4.5 software (Leica Biosystems, Richmond, IL).

\section{DNA methylation array processing}

DNA Extraction and bisulfite conversion of formalinfixed, paraffin-embedded (FFPE) tissue sections was performed as previously described [5]. Briefly, DNA was extracted from FFPE tissue using the Maxwell 16 Plus LEV DNA purification kit (Promega, Madison, WI) according to the manufacturer's instructions. Following bisulfite conversion with the Zymo, EZ DNA Methylation kit (Zymo Research, Irvine, CA), bisulfite DNA was processed using the Illumina Infinium HD FFPE Restore kit (Illumina, San Diego, CA) according to the 
Table 1 Astroblastoma patient demographics and pathology

\begin{tabular}{|c|c|c|c|c|c|c|c|c|c|c|c|}
\hline $\begin{array}{l}\dot{0} \\
Z \\
0 \\
\tilde{U} \\
\tilde{U}\end{array}$ & 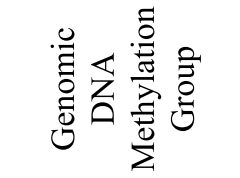 & $\underset{\pi}{\infty}$ & $\frac{\dot{d}}{\stackrel{D}{0}}$ & 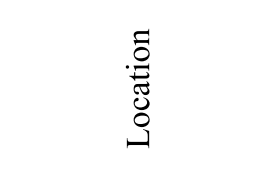 & 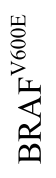 & $\frac{000}{0}$ & $\frac{\frac{\pi}{D}}{\frac{\pi}{Z}}$ & $\begin{array}{l}0 \\
\hat{\sigma} \\
i \\
i\end{array}$ & 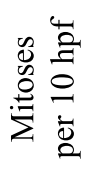 & $\begin{array}{c}\cdot a \\
\tilde{n} \\
0 \\
0 \\
0 \\
z\end{array}$ & 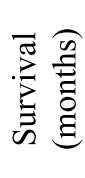 \\
\hline $\mathrm{C} 1$ & EPN/RELA & 10 & $\mathrm{~F}$ & L Parietal & - & Focal + & - & 1 & 0 & - & $143^{*}$ \\
\hline $\mathrm{C} 30$ & EPN/RELA & 19 & $\mathrm{M}$ & L Frontal & - & - & - & 60 & 10 & + & $6^{*}$ \\
\hline $\mathrm{C} 3$ & HGNET MN1 & 16 & $\mathrm{~F}$ & L Parieto-occipital & - & + & + & 20 & 3 & + & $138 *$ \\
\hline $\mathrm{C} 7$ & HGNET MN1 & 33 & $\mathrm{~F}$ & L Temporal & - & + & + & 10 & 3 & + & $120 *$ \\
\hline $\mathrm{C} 10$ & HGNET MN1 & 12 & $\mathrm{~F}$ & R Fronto-parietal & - & + & + & 4 & 0 & - & $147 *$ \\
\hline $\mathrm{C} 13$ & HGNET MN1 & 9 & $\mathrm{~F}$ & R Fronto-parietal & - & + & + & 25 & 14 & + & $68 *$ \\
\hline $\mathrm{C} 29$ & HGNET MN1 & 18 & $\bar{F}$ & L Parietal & - & + & + & 5 & 1 & - & $227 *$ \\
\hline C32 & HGNET MN1 & 12 & $\mathrm{~F}$ & Cerebral & - & + & + & high & 4 & - & ND \\
\hline C33 & HGNET MN1 & 13 & $\mathrm{~F}$ & Parietal & - & - & + & ND & 9 & + & ND \\
\hline C34 & HGNET MN1 & 3 & $\mathrm{~F}$ & Parietal & - & - & + & low & 0 & - & ND \\
\hline C16 & ND & 8 & $\mathrm{M}$ & L Temporal & - & + & + & 0 & 0 & - & $144^{*}$ \\
\hline $\mathrm{C} 21$ & ND & 4 & $\mathrm{~F}$ & L Parieto-occipital & - & + & + & 15 & 3 & + & $131^{*}$ \\
\hline $\mathrm{C} 5$ & PXA & 33 & $\mathrm{M}$ & L Temporal & + & + & - & 15 & 13 & + & 8 \\
\hline C6 & PXA & 33 & $\mathrm{~F}$ & R Frontal & + & + & - & 5 & 2 & - & 59 \\
\hline C9 & PXA & 38 & $\mathrm{~F}$ & R Frontal & + & + & - & 10 & 2 & - & 123 \\
\hline $\mathrm{C} 11$ & PXA & 25 & $\mathrm{~F}$ & R Occipital & + & + & - & 20 & 4 & + & $141^{*}$ \\
\hline $\mathrm{C} 12$ & PXA & 12 & $\mathrm{~F}$ & R Frontal & + & + & - & 17 & 3 & + & 2 \\
\hline $\mathrm{C} 23$ & PXA & 20 & $\mathrm{~F}$ & R Temporal & + & + & - & 22 & 3 & + & $15^{*}$ \\
\hline $\mathrm{C} 24$ & PXA & 20 & $\mathrm{~F}$ & L Parietal & + & + & - & 10 & 2 & - & $79 *$ \\
\hline $\mathrm{C} 22$ & LGG PA/GG & 9 & $\mathrm{M}$ & R Parietal & - & + & - & 15 & 2 & - & 47 \\
\hline $\mathrm{C} 14$ & No Subgroup & 71 & $\mathrm{~F}$ & L Occipital & - & - & - & 4 & 5 & + & 18 \\
\hline C19 & No Subgroup & 36 & $\mathrm{M}$ & R Frontal & - & + & - & 45 & 10 & + & $252 *$ \\
\hline $\mathrm{C} 20$ & No Subgroup & 55 & $\mathrm{M}$ & L Parietal & - & + & - & 25 & 2 & + & 40 \\
\hline $\mathrm{C} 17$ & $\begin{array}{c}\text { Control } \\
\text { Hemisphere }\end{array}$ & 20 & $\mathrm{~F}$ & L Occipital & - & + & - & 10 & 3 & + & $279 *$ \\
\hline C31 & Reactive Cortex & 28 & $\mathrm{~F}$ & R Frontal & - & + & - & 6 & 0 & - & $25^{*}$ \\
\hline $\mathrm{C} 8$ & ND & 40 & $\mathrm{~F}$ & R Temporal & - & + & - & ND & 7 & - & $168 *$ \\
\hline $\mathrm{C} 26$ & ND & 58 & $\mathrm{~F}$ & R Parietal & - & + & - & 0 & 5 & + & 184 \\
\hline
\end{tabular}

Like or similar methylation groups are highlight by the same color

Abbreviations: EPN/RELA ependymoma with RELA fusion, HGNET MN1 high-grade neuroepithelial tumor with MN1 alteration, LGG PA/GG low-grade glioma -

supratentorial pilocytic astrocytoma/ganglioglioma, ND not determined due to inadequate tissue or unavailable follow-up data, $P X A$

pleomorphic xanthoastrocytoma

*The patient was alive at most recent follow-up

manufacturer's protocol. The DNA was then processed using the Illumina Infinium Methylation EPIC BeadChip kit (Illumina) and scanned on the Illumina HiScan system according to the manufacturer's instructions. Beta values representing the fraction of methylated cytosine present at each CpG site were calculated with Illumina Genome Studio software using default settings. DNA methylation data analysis was performed with the statistical programming language $\mathrm{R}$ ( $\mathrm{R}$ Core Team, 2016). Raw data files generated by the iScan array scanner were read and preprocessed using minfi Bioconductor package [2]. With the minfi package, the same preprocessing steps as in Illumina's Genomestudio software were performed. In addition, the following filtering criteria were applied: removal of probes targeting the $\mathrm{X}$ and $\mathrm{Y}$ chromosomes; removal of probes containing-nucleotide polymorphism (dbSNP132 Common) within five base pairs of and including the targeted CpG-site; and removal of probes not mapping uniquely to the human reference genome (hg19), allowing for one mismatch. In total, 395,401 common probes of Illumina $450 \mathrm{~K}$ and EPIC arrays were kept for clustering analysis.

\section{Statistical analysis of DNA methylation}

To determine the genomic DNA methylation pattern subgroup affiliation of our $A B$ samples, we used the reference DNA methylation data published by Capper et al. available from the gene expression omnibus (GSE73801) [5]. Our AB samples were combined with the 2801 reference CNS tumors and control brain tissues for 
unsupervised hierarchical clustering as previously described [5]. In brief, the 32,000 most variable methylated CpG probes measured by standard deviation across combined samples were selected. 1-Pearson correlation was calculated as distance measured between samples and the unsupervised hierarchical clustering was performed by the average linkage agglomeration method. The probe level beta values were also analyzed using t-stochastic neighbor embedding (t-SNE) [20] with the tsne package (version 0.1-3) in R [7]. Hierarchical clustering and t-SNE analyses were repeated using a reduced reference set of tumors $(N=195)$ using the top 10,000 most differentially methylated probes. Supervised analysis was performed using the random forest methylation class prediction algorithm (V11b2) by uploading raw IDAT files to www.molecularneuropathology.org website [5].

\section{Detection of copy number aberrations}

Copy number variation analysis from DNA methylation arrays was performed with the conumee Bioconductor package [11] using default settings. The combined intensities of all available $\mathrm{CpG}$ probes were normalized against control samples from normal brain tissue using a linear regression approach. Mean segment values of -0.18 and 0.18 were used as threshold to call copy number loss and gain, respectively. The control cohort used to evaluate the reference tumors from Capper et al. [5], profiled by $450 \mathrm{~K}$ DNA methylation analysis included all control samples from the dataset $(N=119)$. For copy number analysis of the $\mathrm{AB}$ samples, an alternative control cohort consisting of 26 normal brain samples profiled by the $850 \mathrm{~K}$ array was utilized.

\section{Kaplan-Meier analysis}

Survival analysis was performed by Mantel-Cox log rank test with pairwise comparisons using IBM SPSS Statistics v. 19.0 software. A $P$ value of 0.05 or less was considered statistically significant.

\section{Results}

\section{Genomic DNA methylation}

$850 \mathrm{~K}$ methylation analysis was performed on 23 of the 27 histologically-defined primary $\mathrm{AB}$ cases and 1 recurrent tumor (24 total samples). All but one sample (C21) yielded DNA methylation profiles of sufficient quality for subsequent analysis. Unsupervised hierarchical clustering and t-SNE analysis was performed by comparing the top differentially methylated probes first to a comprehensive reference series consisting of 2801 tumors representing all described DNA methylation classes (Additional file 1: Figure S1) [5]. This analysis was then reduced to a subset of 195 tumors consisting of: HGNET-MN1 $(n=21)$; PXA $(n=44)$; supratentorial ependymoma with C11orf95-RELA fusion (EPN-RELA; $n=70$ ); supratentorial pilocytic astrocytoma and ganglioglioma (LGG-PA/GG-ST; $n=24$ ); control reactive cortex (CONTR-REACT; $n=23$ ); and control cerebral hemisphere (CONTR-HEMI; $n=13$ ) (Fig. 1; Additional file 2: Figure S2). By unsupervised analysis, the ABs failed to cluster into a single group, and instead mostly distributed into previously defined DNA methylation classes. The results from hierarchical clustering and t-SNE analysis were concordant in 20 of 23 samples (Figs. 1 and 2). Eight tumors grouped with HGNET-MN1, seven with PXA, two with EPN-RELA, and one with LGG-PA/GG-ST. Two tumors clustered with either reactive cerebral cortex (C31) or control cerebral hemisphere (C17) (Fig. 1a). The latter was likely due to contamination from normal brain within the sample. An additional three tumors exhibited discordant grouping between the hierarchical clustering and t-SNE analysis. One of these (C19) was discordant between three methylation classes (CONTR-HEMI, CONTR-REACT, and LGG-PA/GG-ST). The other two tumors ( $\mathrm{C} 14$ and $\mathrm{C} 20)$ clustered together by hierarchical clustering, but not within any defined reference methylation class. They did, however, group with PXA by t-SNE analysis (Fig. 1b).

As an additional comparison, we also performed supervised analysis using the www.molecularneuropathology.org website, which employs a random forest methylation class prediction algorithm, using the comprehensive reference set used in the initial unsupervised clustering/t-SNE analysis [5]. For tumors with scores above the threshold values, the supervised analysis was concordant with the unsupervised methods (Fig. 2). Five tumors yielded a probability score below the reporting threshold of 0.90; however, in two of these (C22, and $\mathrm{C} 30$ ), the highest probability was consistent with the unsupervised hierarchical clustering analyses (LGG-PA/GG-ST 0.55 and EPEND RELA 0.89, respectively). Three additional cases (C14, C19, and $\mathrm{C} 20)$ yielded unreliably low probability scores below 0.15 (Additional file 3: Table S1).

\section{Orthogonal validation of DNA methylation groups}

Next, we performed orthogonal molecular analysis to determine if the ABs in specific DNA methylation classes contained the hallmark mutations or gene rearrangements of lesions within those classes. Limited molecular evaluation was also performed on tumors for which insufficient DNA was available for methylation analysis. All eight samples clustering with the HGNET-MN1 methylation class demonstrated evidence of MN1 rearrangement by FISH (Fig. 2; Additional file 4: Figure S3). Support for an MN1 rearrangement was also found by FISH in two additional cases for which insufficient material was available for DNA methylation analysis or for which methylation analysis failed (C16 and C21, respectively). 


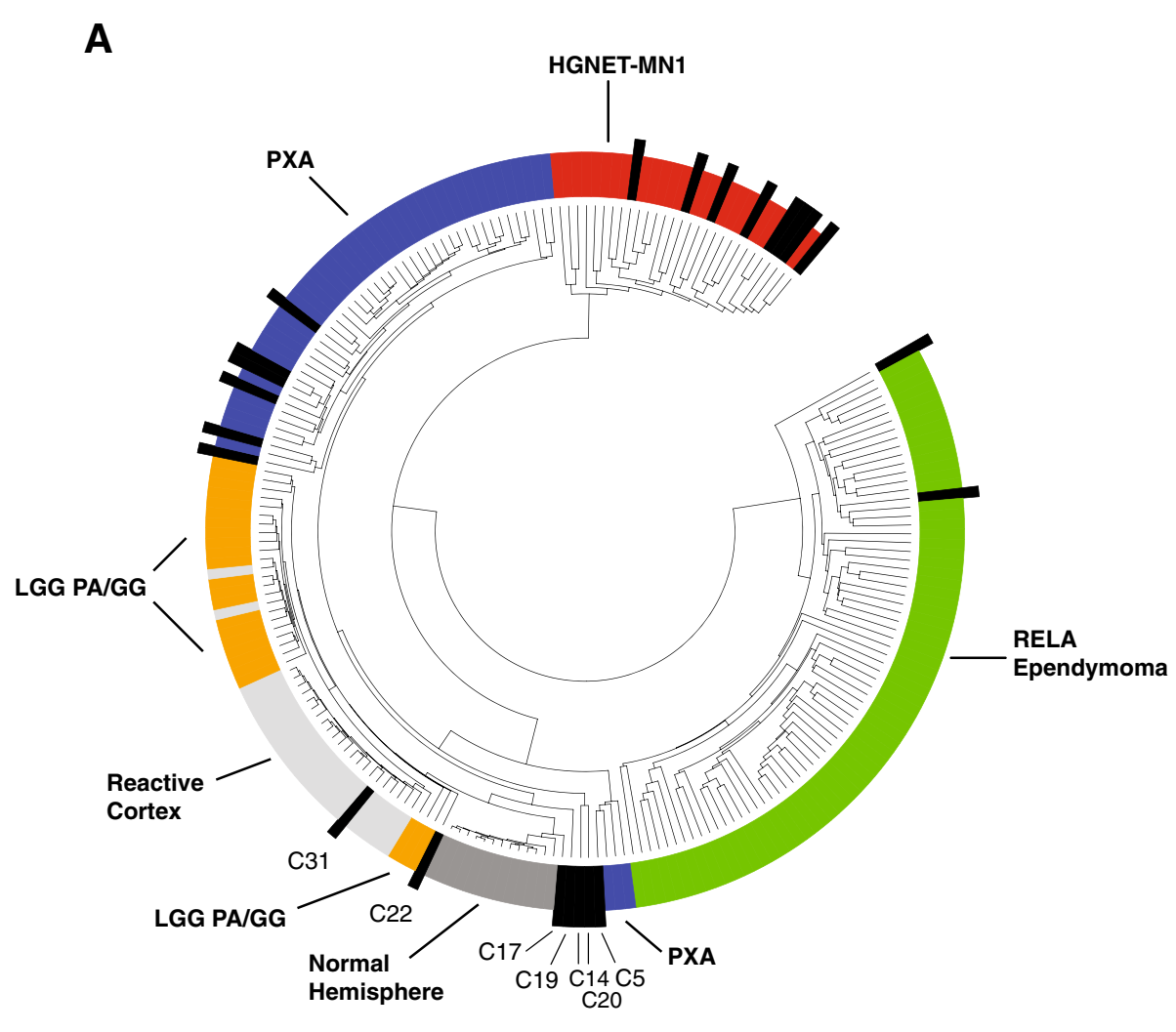

B

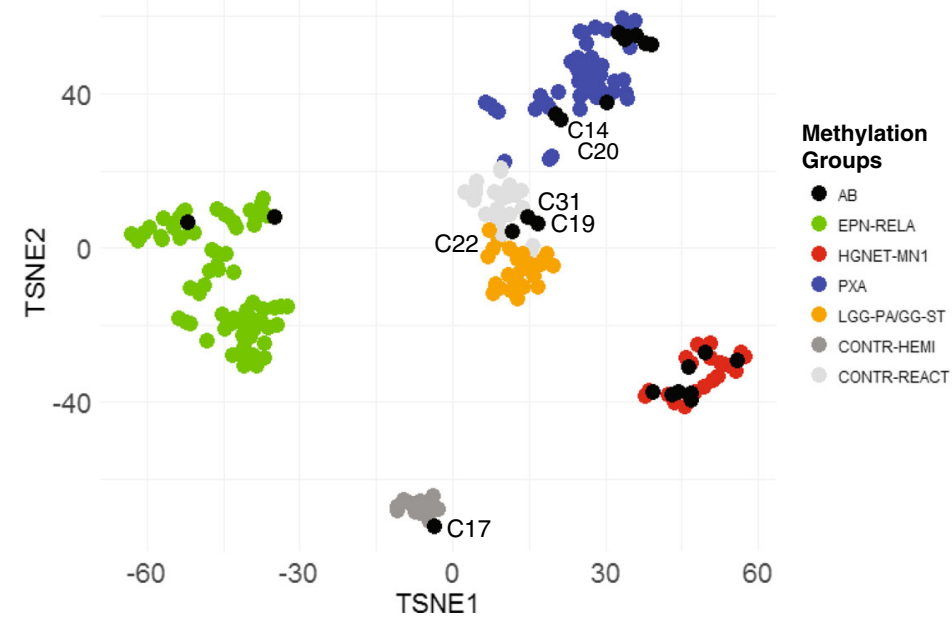

Fig. 1 Histologically-defined ABs do not represent a homogeneous molecular group by DNA methylation profiling. The top 10,000 differentially methylated probes were used to perform unsupervised hierarchical clustering (a) or t-SNE analysis (b) of ABs and a reference group of tumors from selected DNA methylation classes from the Capper et al. dataset [5]

The BRAF V600E mutation was identified in seven of nine tumors clustering in the PXA methylation group by $\mathrm{t}$-SNE (Table 1). BRAF ${ }^{V 600 E}$ mutations were not detected in the two tumors in which t-SNE and hierarchical clustering were discordant (C14 and C20) (Figs. 1 and 2). The two tumors that clustered within the EPN-RELA methylation class were also found to have evidence of RELA rearrangement by interphase FISH.

\section{Chromosomal copy number}

To further evaluate the relationship between $\mathrm{ABs}$ and their respective molecular groups, we evaluated the copy number profiles of $\mathrm{ABs}$ compared to the respective reference tumors. ABs clustering in the HGNET-MN1 group variably demonstrated loss of chromosomes 22q, 14 , and broad regions of X (three of eight cases), similar to previous findings reported in a small cohort of 


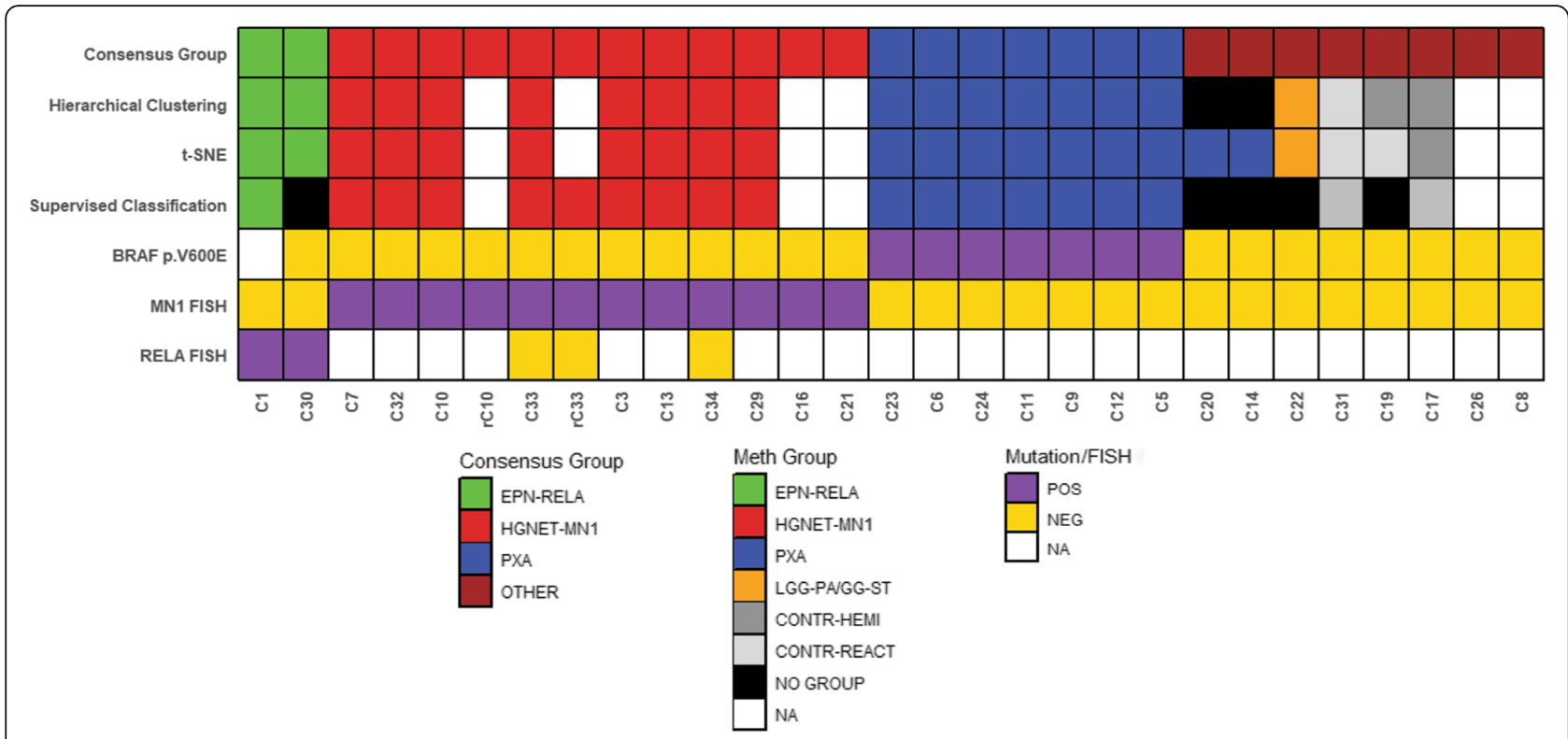

Fig. 2 Orthogonal validation of molecular classification of ABs. Orthogonal testing including DNA methylation profiling, BRAF ${ }^{V 600 E}$ sequencing, and MN1 and RELA FISH was performed on ABs with sufficient material. Supervised analysis was performed using the random forest methylation class prediction algorithm version 11b2 (www.molecularneuropathology.org). Recurrent tumors are indicated by " $\mathrm{r}$ "

MN1-rearranged ABs [10] (Fig. 3a, Additional file 5: Figure S4A). These findings were consistent with those of the reference cohort of HGNET-MN1 tumors, with the exception of a slightly increased proportion of chromosome 14 loss in ABs.

$B R A F^{V 600 E}$-positive ABs that grouped with PXA showed more extensive chromosomal instability compared to other ABs (Fig. 3; Additional file 5: Figure S4A), with frequent gains of chromosome 5, chromosome 7, and chromosome 19; and loss of chromosomes 10,18 , and 6q. Unlike commonly observed in glioblastoma and in the approximately 20\% of PXAs (especially anaplastic PXAs) with chromosome 7 and 10 aberrations [21], gain of chromosome 7 and loss of chromosome 10 were mutually exclusive in ABs (Additional file 5: Figure S4A). While interpretation of data for ABs clustering with EPN-RELA was limited by case number, observed copy number variations were compatible with findings in the reference cohort of RELA ependymomas (Fig. 3c).

Tumors showed few recurrent focal copy number abnormalities within or between DNA methylation classes. Loss of the $C D K N 2 A / B$ locus was observed in one tumor each grouping with HGNET-MN1 and EPN-RELA (Additional file 5: Figure S4B). Three of seven tumors that both grouped with PXA and contained $B R A F^{V 600 E}$ mutations showed focal copy number loss at $C D K N 2 A / B$ (Additional file 5: Figure S4B). Neither $B R A F$ wildtype tumors grouping with PXA in the t-SNE analysis (C14 and C20) showed CDKN2A/B loss, nor did any of the other tumors lacking known driver mutations. No other recurrent focal copy number abnormalities were observed across any of the groups.

\section{Histopathology}

$M N 1$-rearranged, $B R A F^{V 600 E}$-mutant, RELA-rearranged, and tumors without identified driver mutations all occasionally demonstrated nuclear pseudoinclusions [15] (Fig. 4f and h). MN1-rearranged tumors more often showed vascular and/or generalized sclerosis. Three of the 10 MN1-rearranged tumors demonstrated marked sclerosis and contained hyalinized areas consisting of nearly entirely sclerotic vessels as depicted to the right in Fig. 4b. Although the most marked sclerosis was seen in this group, mild vascular sclerosis was also occasionally seen in tumors in the other molecular groups (Fig. 4e, j, and k). $B R A F^{V 600 E}$-mutant $\mathrm{ABs}$ tended to have stouter cells (Fig. 4e-g); however, such cells were also occasionally seen in MN1-rearranged and other tumors (Fig. 4a).

When examining $\mathrm{AB}$ histological features which we previously cataloged [15], RELA-rearranged tumors often showed clear or signet ring-like cells (Fig. 4k and 1); however, these were also observed in select tumors from the other types. Eosinophilic granular body-like structures and spheroid hyaline bodies were absent in RELAand MN1-rearranged tumors, but were common in $B R A F^{V 600 E}$-mutant tumors and tumors without known driver mutations (Fig. 4i and j). Rhabdoid-like cells were not identified in RELA-rearranged tumors, but were found in all other types. All but two MN1-rearranged tumors and both RELA-rearranged tumors lacked lymphocytic infiltrates, 

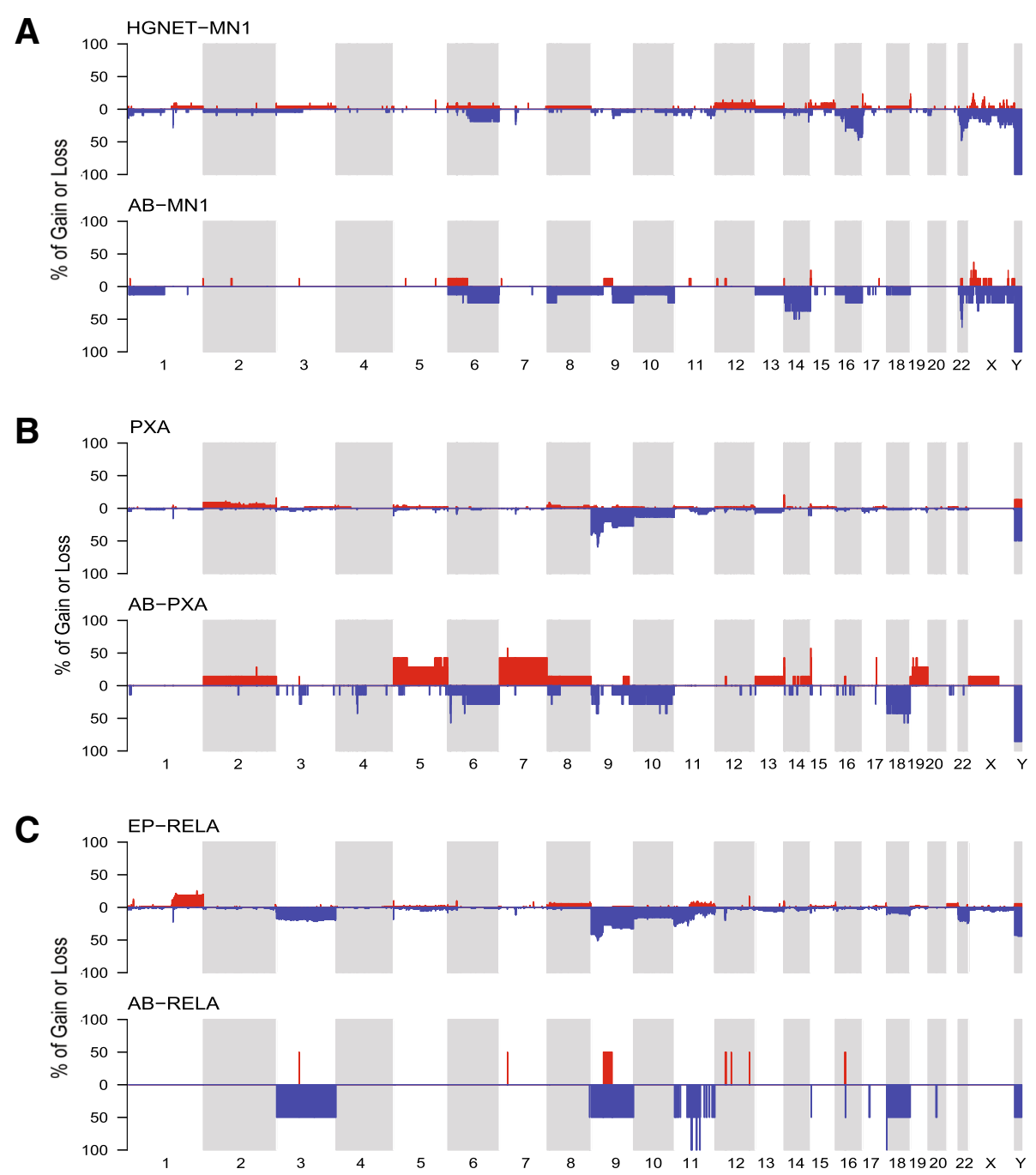

Fig. 3 Chromosomal copy number variations in $A B$ molecular groups compared to reference tumors. Copy number frequency plots were constructed using copy number profiles of reference tumors and ABs grouping with (a) HGNET-MN1, (b) PXA, and (c) EPN-RELA DNA methylation classes

which were common in other types. Multinucleated cells were present in almost all tumors except two $M N 1$-rearranged tumors, the low-grade RELA-rearranged lesion (C1) and one other lesion (C2). Overall, RELA-rearranged tumors tended to lack some histologic features common in most other groups; e.g., lymphocytic infiltrates, eosinophilic granular material, and rhabdoid-like cells.

\section{Clinicopathologic correlates}

Nine of the 10 consensus tumors with $M N 1$ rearrangement $(90 \%)$ presented in female patients aged 3-33 years and 1 was from an 8-year-old male (mean age, 12.8 years; median, 12 years) (Table 1). Eight of 10 MN1-rearranged tumors were olig2 immunopositive, and none showed hypermethylation of the $\mathrm{O}^{6}$-methylguanine methyl transferase (MGMT) gene promoter via the EPIC BeadChip (Table 1). Similarly, six of seven $B R A F^{V 600 E}$-mutant ABs
(87.5\%) occurred in females aged $12-38$ years and one presented in a 33-year-old male patient (mean, 25.9 years; median, 25 years) (Table 1). All were olig2 immunopositive. Two cases (C5 and C9), from patients 33 and 38 years -of-age, respectively, exhibited MGMT promoter hypermethylation, as previously described [15].

RELA rearrangements were detected in tumors from one female and one male ( $\mathrm{C} 1$ and $\mathrm{C} 30)$, aged 10 and 19 years, respectively. Olig2 immunohistochemical staining was equivocal in $\mathrm{C} 1$ and negative in C30. Neither showed MGMT promoter hypermethylation. Six other tumors negative for $B R A F^{V 600 E}$ mutations and $M N 1$ or RELA rearrangements consisted of lesions from three females and three male patients ranging in age from 4 to 71 years. None showed MGMT promoter hypermethylation. All were olig2 immunopositive, except C14 [15]. 


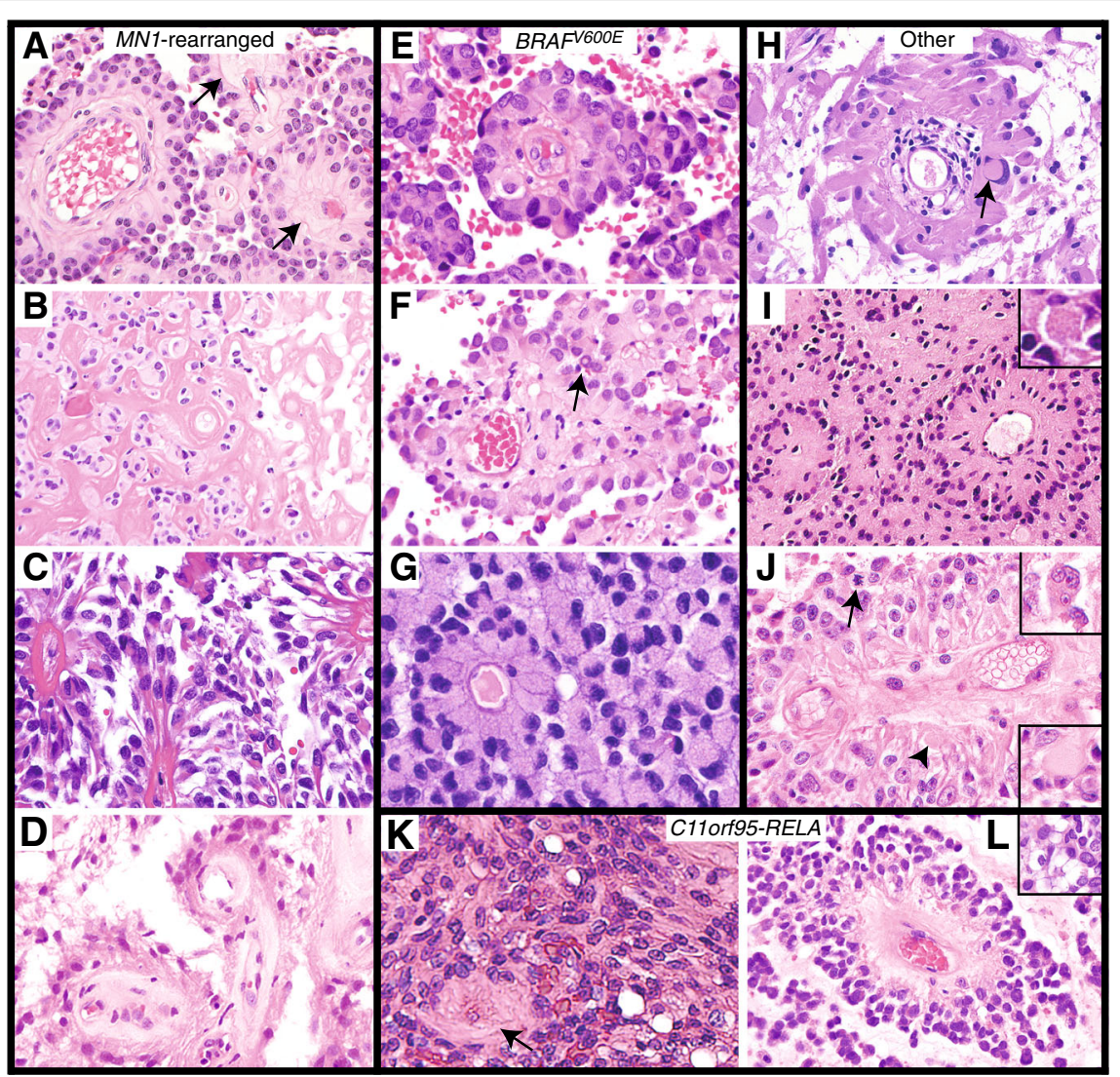

Fig. 4 AB tumor histology. a-d MN1-rearranged tumors: (a) Case C10 showing mild vascular sclerosis (arrows). This image depicts recurrent tumor. b Case 10. The original lesion was highly sclerotic. c Case C3 showed thin tapering process with expanded endfeet in some areas (shown) and stouter clear cells in other areas. Mild vascular sclerosis is again noted. $\mathbf{d}$ Case C16 demonstrating fibrillary areas and vascular sclerosis. e- $\mathbf{g}$ $B R A F^{V 600 E}$-positive cases: (e) Case C12 with mild vascular sclerosis. $\mathbf{f}$ Case C11. A pseudonuclear inclusion is present (arrow). $\mathbf{g}$ Case C6. h-j Cases without known driver mutations: (h) Case 22 showing monopolar columnar-like cells and a pseudonuclear inclusion (arrow). i Case C14. An eosinophilic granular body is shown in the inset. $\mathbf{j}$ Case C19 demonstrating mitotic activity (arrow), free hyaline bodies (arrowhead), intracellular hyaline bodies (lower inset), and multinucleate cells (upper inset). k-I C110rf95-RELA tumors: (k) Case C1, low-grade C110rf95-RELA lesion showing AB histology and mild vascular sclerosis (arrow). I Case C30 demonstrating ependymal-like pseudorosettes and clear signet ring-like cells (inset)

\section{Survival analysis}

Survival analysis was statistically limited by the relatively small number of tumors in each group, but overall showed a significant difference between molecular groups (Mantel-Cox, $P=0.045$; Fig. 5). In pairwise analysis, there was no appreciable difference in overall survival between $\mathrm{ABs}$ with $B R A F^{V 600 E}$ mutations and tumors without specific driver mutations $(P=0.398)$. There did appear to be a difference in survival between MN1-rearranged tumors and tumors without identified driver mutations (other tumors); however, this did not reach statistical significance $(P=0.056)$. There was, however, a clear and significant survival advantage for $M N 1$-rearranged tumors compared to $B R A F^{V 600 E}$-mutant tumors $(P=0.013$; Fig. 5). In fact, all $M N 1$-rearranged tumor patients in the cohort are currently alive, despite multiple tumor recurrences in some cases (Additional file 6: Table S2). Four deaths each occurred in the seven
$B R A F^{V 600 E}$ mutation patients and in the eight patients without known driver mutations (Table 1). The overall survival of the MN1-rearranged tumor patients' ranged from 68 to 221 months (mean, 138 months; $n=7$ ) compared to 2 to 141 months (mean, 61 months; $n=7$ ) for patients whose tumors had $B R A F^{V 600 E}$ mutation, and 18 to 279 months (mean, 127 months; $n=8$ ) for patients with neither genetic alteration.

\section{Discussion}

Studies of limited cohorts have suggested genetic heterogeneity among $\mathrm{AB}$ cases $[3,22]$; whereas, others have argued that $\mathrm{AB}$ is a distinct entity characterized by $M N 1$ rearrangement [10]. Our findings confirm that histologically-defined ABs do not represent a single molecular tumor entity, but instead largely cluster into two known genomic DNA methylation classes: HGNET-MN1 and PXA. 


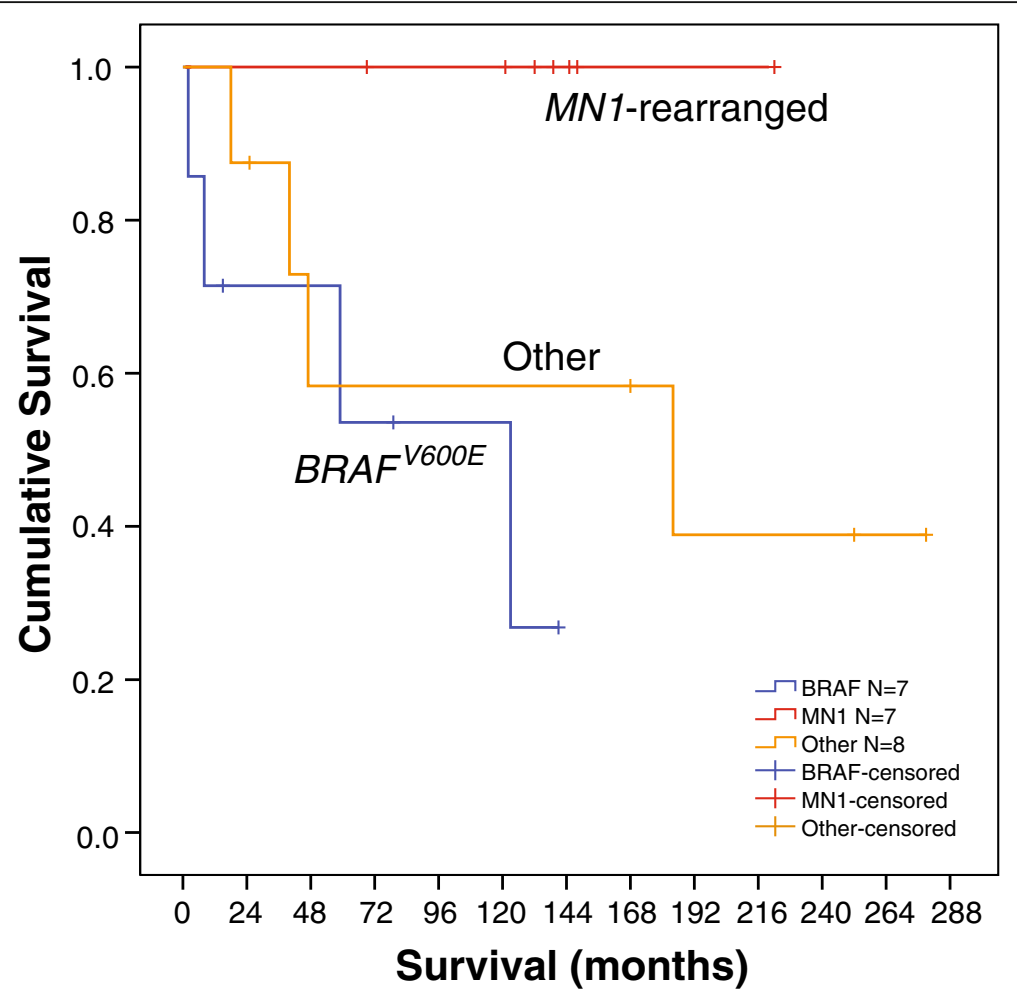

Fig. 5 MN1-rearranged ABs show significantly better survival compared to $B R A F^{V 600 E}$-mutant $A B s$. The initial numbers of patients in each group are indicated in the key. The two RELA/EPEN tumors were not included in the analysis

Tumors designated HGNET-MN1 were previously identified in a cohort of PNETs [19]; however, whether tumors with that designation should be treated as embryonal tumors remains controversial. ABs with $M N 1$ rearrangement presented herein were notable for favorable overall survival. Importantly, they did not demonstrate embryonal features, and were not uniformly high grade, but instead showed a mixture of low- and higher-grade histologies reflected by degree of mitotic activity and the absence or presence of necrosis. These findings suggest that, despite initial discovery in a cohort of tumors diagnosed as PNET, the relationship between histomorphology and clinical behavior in MN1-rearranged tumors requires further evaluation. Given the relatively good overall survival associated with these lesions, a more conservative therapeutic approach may suffice.

The second major molecular group in our AB cohort included tumors grouping with PXA by DNA methylation. While especially $B R A F^{V 600 E}$-mutant $A B$ s showed genomic methylation patterns and other genetic changes common to PXA (e.g., $C D K N 2 A / B$ deletion in three cases), several findings suggest they may not be entirely equivalent entities. For instance, PXAs show a stronger predilection for the temporal lobe, and occur in equal frequency in males and females $[6,12]$. It is, therefore, likely that the PXA reference DNA methylation group is relatively heterogenous compared to other methylation classes and encompasses tumors other than conventional PXAs. This is supported by studies suggesting that other $B R A F^{V 600 E}$-mutant tumors, such as epithelioid glioblastoma, molecularly group with PXA [13].

A small percentage of ABs grouped with RELA ependymomas by genomic DNA methylation analysis. The latter tumors [18] are generally associated with a poorer prognosis [17] and should be distinguished from other $\mathrm{AB}$-like lesions. Thus, AB-like tumors should be investigated for RELA fusion by FISH or screened by immunohistochemistry for p65/RELA and/or L1CAM [8]. RELA ependymomas can be further differentiated from other $\mathrm{AB}$ pseudorosette-predominant lesions by $B R A F$ mutational analysis, FISH for $M N 1$ rearrangement, or genomic DNA methylation analysis [5].

Previous studies have variably argued that some $\mathrm{ABs}$ are related to diffuse astrocytomas. Our data do not support that assertion as we did not identify ABs that molecularly grouped with diffuse astrocytomas. This is likely due to such studies not applying our relatively strict criteria for histopathologic designation of $\mathrm{AB}$ including: requiring that an $\mathrm{AB}$ case demonstrate at least $50 \% \mathrm{AB}$ pseudorosettes and show relative tumor circumscription without evidence of an invasive growth pattern. 
DNA methylation profiling is a powerful tool for tumor classification that can overcome shortcomings of histopathology and more conventional molecular testing, allowing for accurate classification of histologically ambiguous tumors [5, 19]. However, we also found tumors that clustered with no known reference DNA methylation class. Furthermore, Capper et al. [5] described a tumor histologically resembling $\mathrm{AB}$ as unclassifiable by DNA methylation profiling. These findings suggest that additional drivers, other than $M N 1$ rearrangements, $B R A F^{V 600 E}$ mutations, and RELA fusions, may exist for tumors with $\mathrm{AB}$ histology. Expansion of existing tumor methylation reference sets may therefore be necessary to allow classification of such tumors by DNA methylation profiling.

\section{Conclusions}

Regardless of molecular heterogeneity, $\mathrm{AB}$ remains a recognizable histological pattern reflecting tumors with important prognostic and treatment implications, notably their amenability to surgical resection and an overall better prognosis compared to diffuse gliomas. Although survival analysis between molecularly-defined $\mathrm{AB}$ subtypes was limited by sample size, tumors with $M N 1$ rearrangement were characterized by a statistically significant, more favorable outcome compared to BRAF$V{ }^{\prime} 0 O E_{\text {-mutant }} \mathrm{ABs}$, emphasizing the importance of recognizing $\mathrm{AB}$ molecular subtypes for their prognostic and possible treatment implications.

\section{Additional files}

Additional file 1: Figure S1. Unsupervised t-SNE analysis of ABs with the entire Capper et al. dataset [5]. DNA methylation profiles from ABs were analyzed with the comprehensive Capper et al. dataset using the top 32,000 probes. (A). ABs did not group into a single methylation class, but instead grouped within the methylation classes designated (B) EPN-RELA, (C) PXA, (D) HGNET-MN1, (E) CONTR-REACT and LGG-PA/ ST-GG, and (F) CONTR-HEMI. (EPS $5516 \mathrm{~kb}$ )

Additional file 2: Figure S2. Heatmap from unsupervised hierarchical clustering of ABs with selected reference tumors from the Capper et al. dataset [5] using the top 10,000 differentially methylated probes. (PDF $94854 \mathrm{~kb}$ )

Additional file 3: Table S1. Tumor DNA methylation class probabilities from the random forest methylation class prediction algorithm. The lowest probabilities are shaded in blue and the highest probabilities are shaded in red. (XLSX $42 \mathrm{~kb}$ )

Additional file 4: Figure S3. Example images of MN1 FISH. (A) Case C10 showing red and green break apart probes (arrows) and yellow intact MN1 loci (arrowheads). (B) Case C3 with displaced green probe only indicating an unbalanced translocation (arrows). (C) Case C23 showing intact MN1 loci. (D) Case C11 showing multiple intact MN1 loci per nucleus indicating chromosome 22 polyploidy. (EPS $23150 \mathrm{~kb}$ )

Additional file 5: Figure S4. Chromosome copy number analysis. (A) Copy number analysis of individual AB samples. (B) Expanded view of chromosome 9. The consensus DNA methylation groups are annotated by EPN-RELA = "green", HGNET-MN1 = "red", PXA = "blue", and other/unknown $=$ "brown". Recurrent tumor is indicated by "r". (PDF $1754 \mathrm{~kb}$ )

Additional file 6: Table S2. Patient Treatment and Survival Data. (DOCX $25 \mathrm{~kb}$ )

\section{Acknowledgements}

We thank Dr. Werner Paulus of the University of Münster for providing two cases and Michael Kruger for performing statistics for the survival analysis.

\section{Funding}

The study was supported in part by NIH Grant RO1 NS081125 (NLL) and the American Lebanese Syrian Associated Charities (BAO).

\section{Availability of data and materials}

The datasets generated during and/or analyzed during the current study will be made available in the NCBI GEO Datasets repository (GSE125450).

\section{Authors' contributions}

NLL designed and coordinated the study, drafted and revised the manuscript, contributed cases, constructed the case cohort, performed the histological analysis, created and edited figures and analyzed data. AU organized cases and data, performed statistical analyses, obtained and analyzed data and edited the manuscript. SJA performed FISH, methylation arrays and mutational analyses. TL and QTT performed bioinformatic analyses and created figures. BCM, REM, MJS, MMG, MC, MSD, JMR, MAA, CAP and EMH contributed cases and edited the manuscript. BAO designed and coordinated the study, drafted and revised the manuscript, contributed cases, created and edited figures and analyzed data. All authors approved the manuscript.

\section{Ethics approval and consent to participate}

Approval for the study of human tissue was obtained by the Institutional Review Board of each participating institution.

Consent for publication

No patient personal identifying data is included in the study.

\section{Competing interests}

The authors declare that they have no competing interests.

\section{Publisher's Note}

Springer Nature remains neutral with regard to jurisdictional claims in published maps and institutional affiliations.

\section{Author details}

${ }^{1}$ Department of Pathology and Laboratory Medicine, University of Louisville, Louisville, KY 40292, USA. ${ }^{2}$ Department of Biomedical Informatics, University of Arkansas for Medical Sciences, Little Rock, AR 72205, USA. ${ }^{3}$ Department of Biostatistics, St. Jude Children's Research Hospital, Memphis, TN 38105, USA. ${ }^{4}$ Department of Pathology, St. Jude Children's Research Hospital, 262 Danny Thomas Place, Memphis, TN 38105, USA. ${ }^{5}$ Department of Pathology, Vanderbilt University School of Medicine, Nashville, TN 37232-2561, USA. ${ }^{6}$ Departments of Pathology, Duke University Medical Center, Durham, NC 27710, USA. ${ }^{7}$ Department of Pathology, Emory University, Atlanta, GA 30322 , USA. ${ }^{8}$ Department of Pathology, Louisiana State University Health Shreveport, Shreveport, LA 71103, USA. ${ }^{9}$ Department of Pathology, Case Western Reserve University, Cleveland, OH 44106, USA. ${ }^{10}$ Department of Pathology, Beaumont Hospital, Royal Oak, MI 4807, USA. "1'Department of Pathology, The University of Texas Southwestern, Dallas, TX 75390, USA. ${ }^{12}$ Department of Pathology, Microbiology and Forensic Medicine, University of Jordan, Amman, Jordan.

${ }^{13}$ Department of Pathology, University of Utah, Salt Lake City, UT 84112, USA.

Received: 21 January 2019 Accepted: 25 February 2019

Published online: 15 March 2019

References

1. Aldape KD, Rosenblum MK (2016) Astroblastoma. In: Louis DN, Ohgaki H, Wiestler $\mathrm{OD}$, Cavenee WK (eds) WHO classification of tumours of the central nervous system. International Agency for Research on Cancer, Lyon, France, pp 121-122

2. Aryee MJ, Jaffe AE, Corrada-Bravo H, Ladd-Acosta C, Feinberg AP, Hansen KD, Irizarry RA (2014) Minfi: a flexible and comprehensive Bioconductor package for the analysis of Infinium DNA methylation microarrays. Bioinformatics 30:1363-1369. https://doi.org/10.1093/bioinformatics/btu049

3. Bale TA, Abedalthagafi M, Bi WL, Kang YJ, Merrill P, Dunn IF, Dubuc A, Charbonneau SK, Brown L, Ligon AH et al (2016) Genomic characterization of recurrent high-grade astroblastoma. Cancer Genet 209:321-330. https:// doi.org/10.1016/j.cancergen.2016.06.002 
4. Broniscer A, Chamdine O, Hwang S, Lin T, Pounds S, Onar-Thomas A, Shurtleff S, Allen S, Gajjar A, Northcott P et al (2016) Gliomatosis cerebri in children shares molecular characteristics with other pediatric gliomas. Acta Neuropathol 131:299-307. https://doi.org/10.1007/s00401-015-1532-y

5. Capper D, Jones DTW, Sill M, Hovestadt V, Schrimpf D, Sturm D, Koelsche C, Sahm F, Chavez L, Reuss DE et al (2018) DNA methylation-based classification of central nervous system tumours. Nature 555:469-474. https://doi.org/10.1038/nature26000

6. Dias-Santagata D, Lam Q, Vernovsky K, Vena N, Lennerz JK, Borger DR, Batchelor TT, Ligon KL, lafrate AJ, Ligon AH et al (2011) BRAF V600E mutations are common in pleomorphic xanthoastrocytoma: diagnostic and therapeutic implications. PLoS One 6:e17948. https://doi.org/10.1371/journal. pone.0017948

7. Donaldson J (2016) tsne: T-distributed stochastic neighbor embedding for $\mathrm{R}$ (t-SNE). R package version 0.1-3. https://CRAN.R-project.org/package=tsne

8. Gessi M, Giagnacovo M, Modena P, Elefante G, Gianno F, Buttarelli FR, Arcella A, Donofrio V, Diomedi Camassei F, Nozza P et al (2017) Role of immunohistochemistry in the identification of supratentorial C11ORF95RELA fused ependymoma in routine neuropathology. Am J Surg Pathol. https://doi.org/10.1097/PAS.0000000000000979

9. Heuser M, Argiropoulos B, Kuchenbauer F, Yung E, Piper J, Fung S, Schlenk RF, Dohner K, Hinrichsen T, Rudolph C et al (2007) MN1 overexpression induces acute myeloid leukemia in mice and predicts ATRA resistance in patients with AML. Blood 110:1639-1647. https://doi.org/10.1182/blood2007-03-080523

10. Hirose T, Nobusawa S, Sugiyama K, Amatya VJ, Fujimoto N, Sasaki A, Mikami Y, Kakita A, Tanaka S, Yokoo H (2017) Astroblastoma: a distinct tumor entity characterized by alterations of the $\mathrm{X}$ chromosome and MN1 rearrangement. Brain Pathol. [Epub ahead of print]. https://doi.org/10.1111/bpa.12565

11. Hovestadt V, Zapatka M (2015) conumee: enhanced copy-number variation analysis using Illumina DNA methylation arrays. R package version 1.9.0, http://bioconductor.org/packages/conumee/

12. Ida CM, Rodriguez FJ, Burger PC, Caron AA, Jenkins SM, Spears GM, Aranguren DL, Lachance DH, Giannini C (2015) Pleomorphic xanthoastrocytoma: natural history and long-term follow-up. Brain Pathol 25:575-586. https://doi.org/10.1111/bpa.12217

13. Korshunov A, Chavez L, Sharma T, Ryzhova M, Schrimpf D, Stichel D, Capper D, Sturm D, Kool M, Habel A et al (2017) Epithelioid glioblastomas stratify into established diagnostic subsets upon integrated molecular analysis. Brain Pathol. [Epub ahead of print]. https://doi.org/10.1111/bpa.12566

14. Lehman NL (2008) Central nervous system tumors with ependymal features: a broadened spectrum of primarily ependymal differentiation? J Neuropathol Exp Neurol 67:177-188. https://doi.org/10.1097/NEN. 0b013e31816543a6

15. Lehman NL, Hattab EM, Mobley BC, Usubalieva A, Schniederjan MJ, McLendon RE, Paulus W, Rushing EJ, Georgescu MM, Couce M et al (2017) Morphological and molecular features of astroblastoma, including BRAFV600E mutations, suggest an ontological relationship to other corticalbased gliomas of children and young adults. Neuro-Oncology 19:31-42. https://doi.org/10.1093/neuonc/now118

16. Lekanne Deprez RH, Riegman PH, Groen NA, Warringa UL, van Biezen NA Molijn AC, Bootsma D, de Jong PJ, Menon AG, Kley NA et al (1995) Cloning and characterization of MN1, a gene from chromosome 22q11, which is disrupted by a balanced translocation in a meningioma. Oncogene 10: $1521-1528$

17. Pajtler KW, Witt H, Sill M, Jones DT, Hovestadt V, Kratochwil F, Wani K, Tatevossian R, Punchihewa C, Johann P et al (2015) Molecular classification of ependymal tumors across all CNS compartments, histopathological grades, and age groups. Cancer Cell 27:728-743. https://doi.org/10.1016/j. ccell.2015.04.002

18. Parker M, Mohankumar KM, Punchihewa C, Weinlich R, Dalton JD, Li Y, Lee R, Tatevossian RG, Phoenix TN, Thiruvenkatam R et al (2014) C11orf95-RELA fusions drive oncogenic NF-kappaB signalling in ependymoma. Nature 506: 451-455. https://doi.org/10.1038/nature13109

19. Sturm D, Orr BA, Toprak UH, Hovestadt V, Jones DTW, Capper D, Sill M, Buchhalter I, Northcott PA, Leis I et al (2016) New brain tumor entities emerge from molecular classification of CNS-PNETs. Cell 164:1060-1072. https://doi.org/10.1016/j.cell.2016.01.015

20. van der Matten L, Hinton G (2008) Visualizing high-dimensional data using t-SNE. J Mach Learn Res 9:2579-2605
21. Vaubel RA, Caron AA, Yamada S, Decker PA, Eckel Passow JE, Rodriguez FJ, Nageswara Rao AA, Lachance D, Parney I, Jenkins R et al (2018) Recurrent copy number alterations in low-grade and anaplastic pleomorphic xanthoastrocytoma with and without BRAF V600E mutation. Brain Pathol 28: 172-182. https://doi.org/10.1111/bpa.12495

22. Wood MD, Tihan T, Perry AJ, Chacko G, Turner C, Pu C, Payne C, Yu A, Bannykh S, Solomon DA (2017) Multimodal molecular analysis of astroblastoma enables reclassification of most cases into more specific molecular entities. Brain Pathol. [Epub ahead of print]. https://doi.org/10. 1111/bpa.12561

\section{Ready to submit your research? Choose BMC and benefit from:}

- fast, convenient online submission

- thorough peer review by experienced researchers in your field

- rapid publication on acceptance

- support for research data, including large and complex data types

- gold Open Access which fosters wider collaboration and increased citations

- maximum visibility for your research: over $100 \mathrm{M}$ website views per year

At BMC, research is always in progress.

Learn more biomedcentral.com/submissions 